\title{
Experimental and Numerical Studies of Liquid Dispersal from a Soft Projectile Impacting a Wall
}

\author{
Ari Silde $^{1)}$, Simo Hostikka ${ }^{1)}$, Ari Kankkunen ${ }^{2)}$, Juhani Hyvärinen $^{3)}$ and Ilkka Hakola ${ }^{1)}$ \\ 1) Technical Research Centre of Finland (VTT) \\ 2) Helsinki University of Technology (TKK) \\ 3) Radiation and Nuclear Safety Authority (STUK), Finland
}

\section{ABSTRACT}

Liquid release and spread as a consequence of impact of missile on a wall are of interest for the determination of consequences of an airplane crash on a structure. These phenomena have been studied in medium-scale IMPACT tests at the Technical Research Centre of Finland (VTT). In these tests, deformable cylindrical steel or aluminium projectiles impacted a solid concrete wall or a steel force plate. Some of the tests were conducted using a fluid filled ("wet") projectile. The length of the wet projectiles ranged from 0.5 to $1.5 \mathrm{~m}$, the water mass inside the projectile from 15 to $68 \mathrm{~kg}$ and the impact velocity from 70 to $177 \mathrm{~m} / \mathrm{s}$.

This paper concentrates on the methods applied during the impact tests to measure the liquid dispersal phenomena. The main results of preliminary simulations of liquid spread are also presented.

So far, the main parameters of the liquid phenomena measured in the experiments are the velocity and direction of the liquid front coming out from the ruptured projectile, water pooling area on the floor, extent of liquid dispersal far from the target, and drop size of the liquid spray. The velocity and direction of the front of ejected liquid within an approximately 2 $\mathrm{m}$ distance from the target was measured using high-speed (1000 fps) video cameras. In addition, normal DV cameras were located around the target to detect the angle and direction of liquid spread, a general view of the liquid spray, and the average velocity of the liquid front up to approximately $5 \mathrm{~m}$ from the target. The water-pooling area was detected using a measuring grid drawn on the floor and photographing the wet areas. The extent of dispersal of liquid spray far from the impact target was measured with the pure collection trays (steel plates located on the floor). Oil-coated trays were also used to capture the deposited droplets and to measure the drop size using the macro photography technique and proper image analysis software. Specific arrangements to photograph the size and velocity of airborne droplets in the vicinity of the impact target were also developed. This system consists of both a high-speed (1000 fps) and a high-shutter-speed $(\mathrm{t}=1$ $\mu \mathrm{s})$ camera and a stroboscope light (flash time $1 \mu \mathrm{s}$ ) for backward illumination.

The preliminary simulations of liquid dispersal were made using the 3-D Fire Dynamics Simulator code (FDS). The main objective of the analyses was to assess the usability of the code for the simulation of the two-phase flows involving high-speed droplets and to support the experimental work by providing an initial estimate of the spray behaviour. The simulation results indicated that FDS is a tool usable in simulating this kind of behaviour provided that the initial conditions of air speed, angle of droplet release, droplet size distribution, and initial droplet speed are specified. Given these, the formation of the water cloud and the final extent of liquid dispersal can be predicted by FDS reasonably well. Future work with the FDS program should include a validation of the some sub-models. Also, some IMPACT tests using the real boundary conditions will be simulated.

\section{INTRODUCTION}

Fuel dispersal from impacted projectile is of interest for the determination of fuel spread and fire risk following an airplane crash on a structure, and for the validation of the applied simulation techniques. Because very little representative and experimental information can be found from the literature, a medium-scale impact test programme has been initiated by the Technical Research Centre of Finland (VTT) [1], [2], [3] , [4]. See [2] for a detailed description of the test facility.

The tests have covered deformation and breakup of soft missiles impacting a wall and the relating failure mechanisms of the concrete wall. The tests also enable the study of liquid release and dispersal from a ruptured high-speed projectile, since some of the tests are conducted with a fluid filled ("wet") missile [5]. In the wet missile tests conducted so far, steel or aluminium cylinder deformable projectiles impacted a solid concrete wall or a steel force plate. The length of the wet projectiles ranged from 0.5 to $1.5 \mathrm{~m}$, the water mass inside the projectile from 15 to $68 \mathrm{~kg}$ and the impact velocity from 70 to $177 \mathrm{~m} / \mathrm{s}$.

This paper concentrates on the methods used to measure the liquid dispersal phenomena in the impact tests conducted at VTT. The main results of the preliminary simulations of liquid spread using the Fire Dynamic Simulator (FDS) computer program are also presented. 


\section{METHODS USED TO MEASURE THE LIQUID DISPERSAL}

The measured parameters relating to liquid dispersal were the speed and direction of liquid release, extent of liquid spray and liquid pooling area on the floor, and size of deposited and airborne droplets of liquid spray. In spite of the fact that none of the parameters were studied systematically in any of the wet missile tests, the experimental results have given new and valuable information on liquid speed and dispersal phenomena under impact conditions. This information can be utilised in understanding better the fuel dispersal under aircraft impact conditions, and for validation of analysing methods for the determination of fuel spread and fire risk following an airplane crash.

\section{Discharge Velocity and Direction of Liquid Release}

The discharge velocity and direction of liquid release spurting out from the projectile were measured from the still figures taken by the high-speed (1000 fps) and normal-speed (25 fps, 50 field/s) video cameras (Fig. 1). The high-speed cameras were used to study the momentary speed close to the impact target, i.e. within a $1-2 \mathrm{~m}$ distance from the missile (Fig. 1 (a)), whereas the normal-speed DV cameras located in different places around the target were only applicable for the general overview and for a rough determination of the average speed of the liquid front farther from the target (Fig. 1 (b)). The position of the visible front of liquid was determined from each still figure of the video cameras, taking into account the exposure time and uncertainty limits of the measurements. Also, some geometrical corrections had to be made due to the angle between the lines of camera focus and travel of missile. Because the time interval (frame rate) between the sequential figures was known, the speed of the liquid front could be estimated within this method.
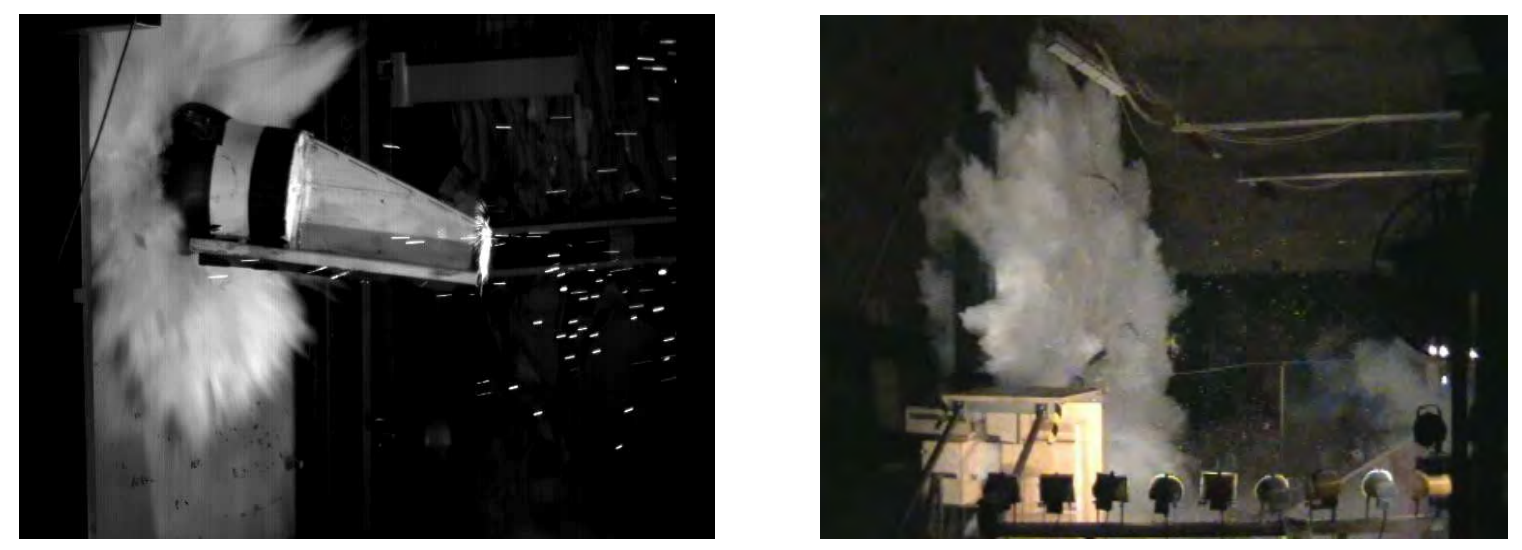

Fig. 1. Example of a still figure taken of a high-speed video camera (a) and a normal-speed DV camera (b).

\section{Water-Pooling Area}

A visibly wet area (pool) formed on the floor near the impact target due to deposited droplets and the liquid spill from the missile. Part of the liquid was also splashed off from the surrounding structures. The pool area was estimated by means of a measuring grid drawn on the floor in front of the impact target (Fig. 2). The wet areas were photographed by digital and DV cameras soon after the tests. In some tests, the collection trays (steel plates) were also located on the floor for a rough estimation of the average amount of water per area on the trays. Unfortunately, the floor dried very fast after the tests due to the heating effect of the lamps. This caused some uncertainties in the comparison of the results between different tests, because the photography could not be conducted at exactly the same time after each test. 


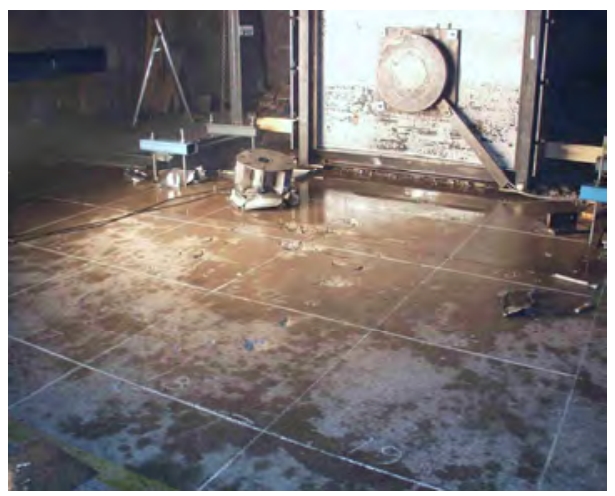

Fig. 2. Example of the wetted area on the floor after an impact test.

\section{Extent of Dispersal of Liquid spray}

A part of liquid spray was dispersed relatively far from the impact target where the water concentration was so small that no visible and uniform pool was formed on the floor. At that distance, the extent of liquid dispersal was assessed using the pure collection trays (Fig. 3). The trays were mostly located in two parallel lines directly sideward, up to the distance of $15 \mathrm{~m}$ from the impact target. Using proper illumination, deposited droplets could clearly be seen on the trays (Fig. 4). Very small droplets cannot be detected using this method. Neither is the drop size measurement reliable. However, this method was usable for the estimation of the extent of spray dispersal, i.e. how far away the medium- and large-size droplets were maximally travelling from the impact target.

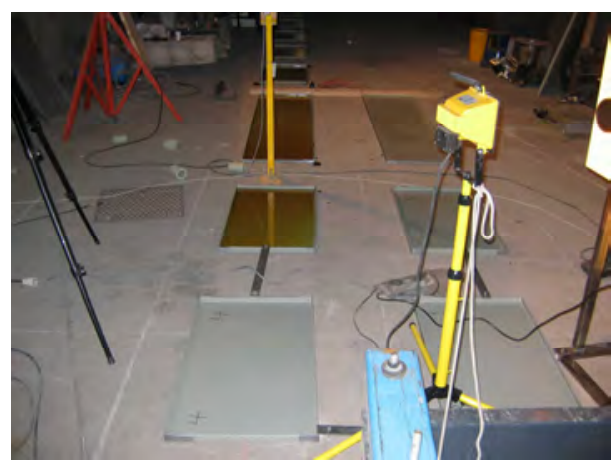

Fig. 3. The collection trays on the floor.

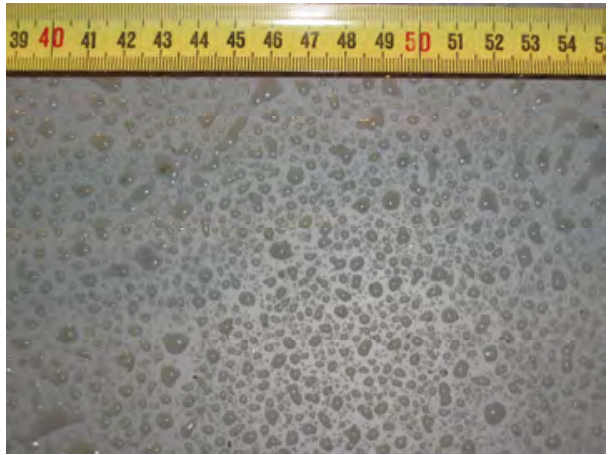

Fig. 4. Liquid splashes (droplets) on a collection tray. 


\section{Size of Deposited Droplets}

Some of the collection trays were coated by a 1-2 mm thick, high-viscosity oil layer to capture the deposited droplets and enable the drop-size imaging and determination (Fig. 5). The density of the oil was lower than that of water to intensify the sinking of drops into the oil layer. The droplet size in the oil layer was measured with specific image analysis software. This method is not suitable very close to the impact target because of high droplet density and velocity. A few meters away from the target, the method will give information about droplet size and water distribution (concentration) on the measurement location. The earlier laboratory studies indicated that water droplets may slowly coalesce in oil even if the viscosity of the oil is very high. In addition, very small droplets may evaporate before they penetrate into the oil layer. This is why a number of small droplets were probably underestimated in the oil samples. Also, water droplets were probably splashed off the surrounding structures and impinged on the collection trays, affecting the size distribution on the trays. It is worth mentioning that the size of droplets detected on the oil-coated trays does not represent necessarily the size of airborne droplets of the "primary" liquid spray. The smallest droplet diameter that could be detected within this method was around $15 \mu \mathrm{m}$.

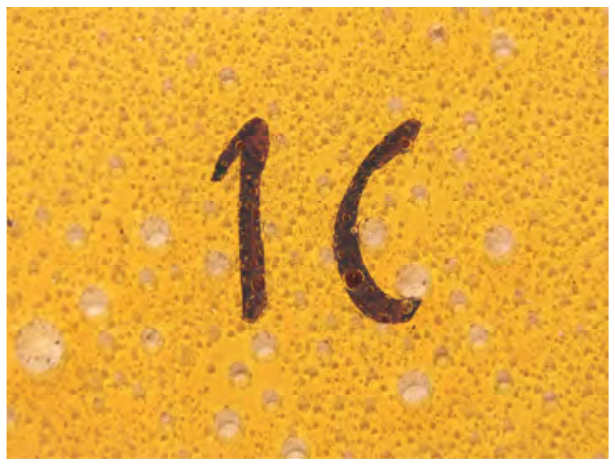

Fig. 5. Liquid droplets in oil layer on a collection tray.

\section{Size and Velocity of Airborne Droplets}

The drop size is a vital input parameter for many numerical methods that can be applied in assessing the liquid dispersal and combustion, especially for those that do not contain any modelling approach for droplet breakup. The drop size has influence on, for example, the extent of spray dispersal, evaporation of droplets, and the flammability and combustion mode of the fuel droplet cloud. Measurement of the size of airborne droplets of high-velocity spray under very energetic and vigorous impact conditions is a very challenging task. Use of several "sophisticated" methods such as phaseDoppler anemometry, Malvern particle size analyzer, or Particle Imaging Velocimetry (PIV) was considered in the project. Although they were available, use of these methods proved to be very time-consuming and expensive, and their assembly and protection needed very careful planning and complex construction. Also, the obtainable information on these methods was considered not to be enough compared to the difficulties and risks to be overcome. After careful consideration, it was decided to use a specific video camera system to measure the size and velocity of airborne droplets. A schematic picture of the measuring system used in selected impact tests is illustrated in Fig. 6. The assembly of the system was technically feasible and the system was supposed to be cost-effective because the system could give information on droplet size, velocity and density. The system consists of two different, parallel cameras with a proper backlighting illumination: a high-speed camera and a high-shutter-speed camera. A high-speed camera (500 fps) was used for drop-size measurement. The camera was synchronised with a high-speed stroboscope to enable an exposure time of $1 \mu$ s that "stopped" the motion of single drops. A high-shutter-speed camera (25 fps) with double-pulse imaging located parallel to the high-speed camera was used for the velocity measurement of drops in the focus area. The time difference between double images was $50 \mu \mathrm{s}$. A DC-based lamp was used for backlighting illumination for the high-shutter-speed camera. 


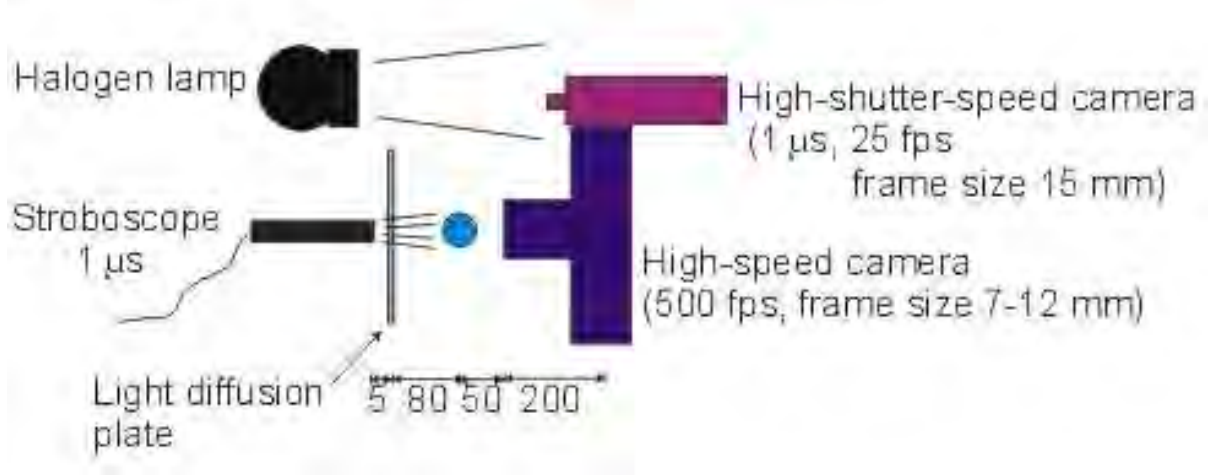

Fig. 6. Size and velocity measurement of airborne droplets.

\section{GENERAL EXPERIMENTAL FINDINGS ON LIQUID DISPERSAL}

The deceleration of the missile is very rapid on impact and the liquid spurts out from the missile when the liquid container fails, leading to dispersal around the target. The liquid release started along the surface quite perpendicularly to the missile direction, and formed a fairly "flat" and circular splash pattern. However, in some experiments, the splash pattern was partly asymmetric, because the fragments of the missile shell and the impact angle of the missile affected the liquid propagation. The pattern became thicker as the breakup of the liquid core began. The initial discharge speed of the liquid front coming out from the ruptured missile was mostly higher than the impact velocity of the missile, but the propagation speed of the spray front decreased rapidly when the distance from the source increased.

\section{PRELIMINARY SIMULATIONS OF LIQUID SPRAY}

The spread of liquid fuel as a result of an intentional or accidental aircraft crash may cause a sudden fire with hazardous effects on the safety of the NPP. For the assessment of such effects, numerical simulation of spreading of liquid droplet cloud is needed. The main problem of the simulations is the definition of the boundary conditions for the liquid phase: initial drop-size, velocity and spreading angle. Additionally, the computer codes to simulate the liquid/droplet dispersal need to be validated.

The preliminary simulations of liquid spray as a consequence of the impact of a fluid-filled missile on a solid obstruction were conducted using the Fire Dynamics Simulator (FDS) program [6]. FDS is a Navier-Stokes solver design for the simulation of fires and the corresponding heat and mass transport processes. FDS uses the Large Eddy Simulation (LES) technique to model the gas-phase turbulence and Lagrangian particles to account for the two-phase flows. Until now, FDS has no model for droplet breakup due to aerodynamic forces, but the needs of implementation of such a model will be considered in the future.

The main purpose of the simulations was to asses the usability of the FDS program for the simulation of the two-phase flows involving high-speed droplets and to support the experimental work by providing an initial estimate of the spray behaviour. The aim was also to find the sensitivity to the assumptions concerning the initial speed, direction and the size distribution of the water droplets, and some numerical parameters (in particular related to inter-phase friction).

The simulated domain was $12 \mathrm{~m}$ long (x), $10 \mathrm{~m}$ wide (y) and $5 \mathrm{~m}$ high (z). The target wall was located $2.0 \mathrm{~m}$ from the domain boundary. The flying missile was not included in the model, but its effect on the airflow field was simulated by the air impulse. The droplets were released from a virtual ball surface centred at the distance of 0.1 from the wall. The initial speed of the air impulse ranged from 100 to $200 \mathrm{~m} / \mathrm{s}$ and the initial droplet speed from 100 to $300 \mathrm{~m} / \mathrm{s}$. Both ends of the domain were open and the ceiling, floor and the sidewalls were solid, except in one simulation, where one of the sidewalls was assumed to be open.

An example of the simulated spread pattern of the liquid spray in a vertical plane cutting through the virtual droplet source perpendicular to the missile direction is illustrated in Fig 7. The first six figures are taken with $20 \mathrm{~ms}$ intervals, starting at $80 \mathrm{~ms}$ from the beginning of the simulation. The last two figures are for $0.4 \mathrm{~s}$ and $0.8 \mathrm{~s}$, showing how the water hits the ceiling and starts to drop down due to gravity. 

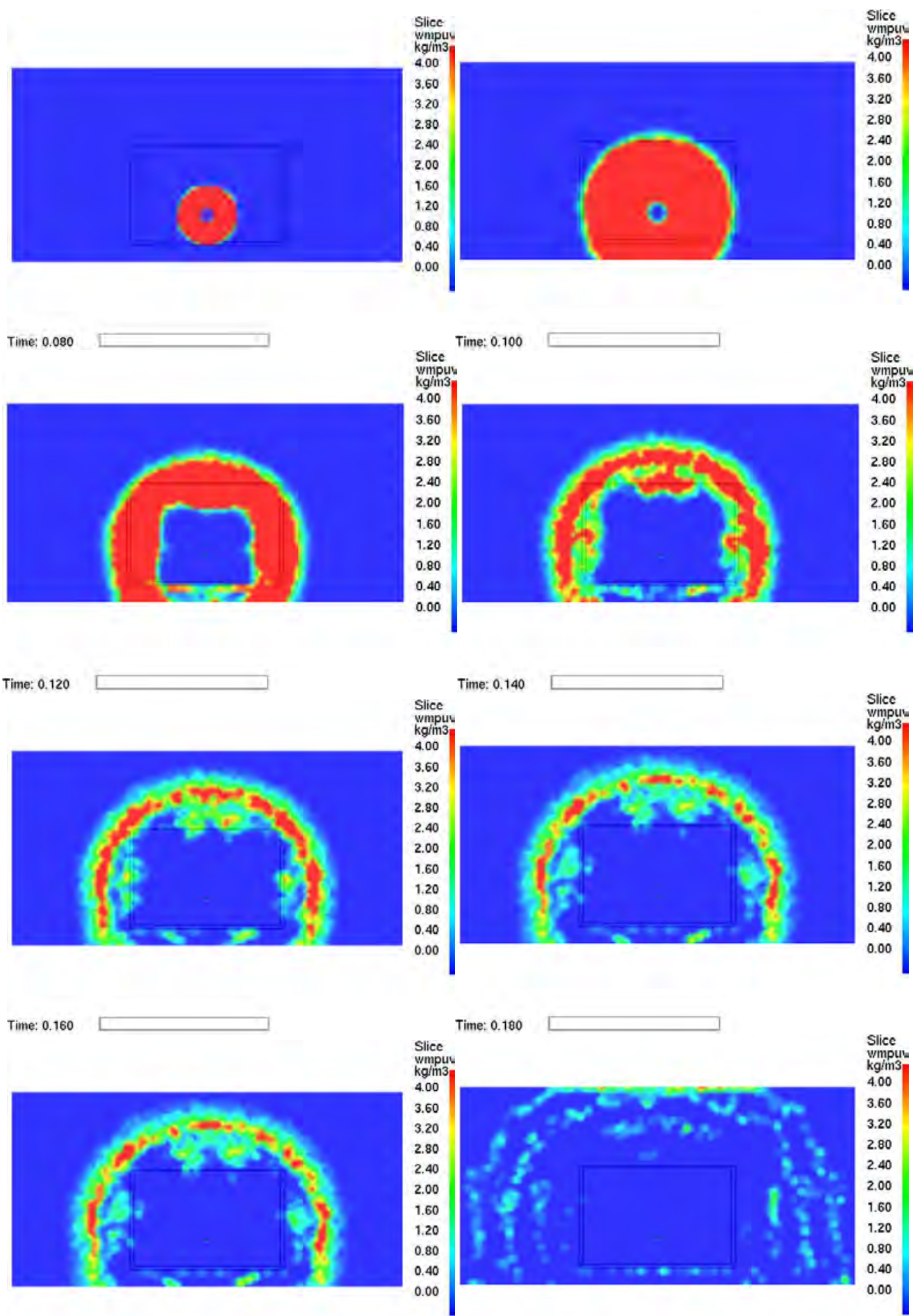

Fig. 7. Simulated spread pattern of the liquid spray. 
The simulation results indicated that if the water spray is released to still air, the splash is very symmetric and only slightly wider in the direction perpendicular to the missile path (y-direction). The airflow tends to flatten the splash region. At higher initial mean diameters, the spray can spread longer in the initial direction of release, due to the higher momentum of the large droplets. The higher initial speed of droplets results in a slightly wider spreading pattern. However, the effect is clearly smaller than the effect of the initial angle of droplet release.

An example of the simulation of speed of spray propagation for different values of the numerical parameter (FL) limiting the momentum transfer between the liquid and gas phases is shown in Fig. 8. Close to the surface where the spray is released, the progress speed is of the same order as with the initial velocity of $150 \mathrm{~m} / \mathrm{s}$. The propagation speed decreases rapidly when the distance from the source increases; at $2 \mathrm{~m}$ the speed is about $50 \mathrm{~m} / \mathrm{s}$ and at $4 \mathrm{~m}$ it is in the range $10 \ldots 20$ $\mathrm{m} / \mathrm{s}$.

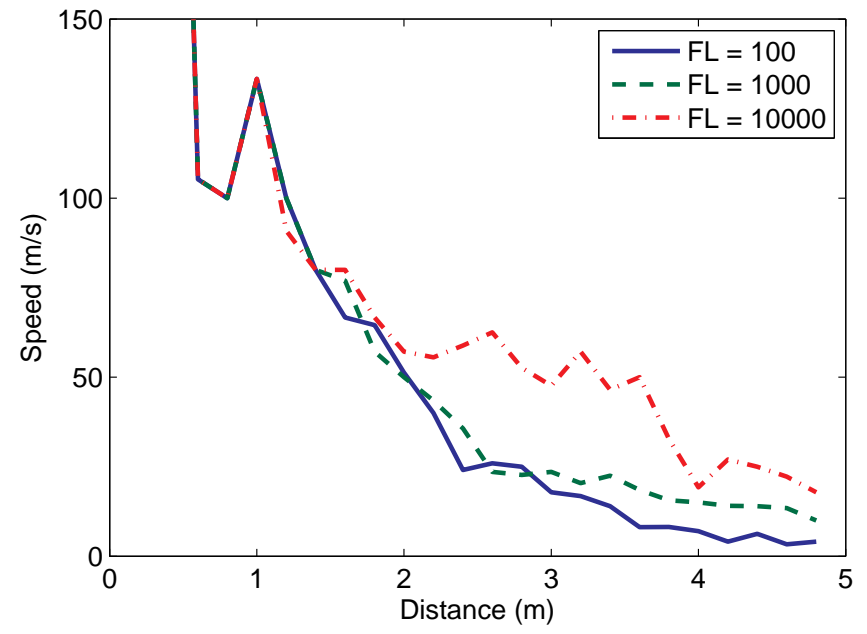

Fig. 8. Speed of spray propagation at different values of the numerical parameter.

Generally speaking, the simulation results indicated that the FDS program is a tool usable in simulating the behaviour of high-velocity liquid spray formed on impact. The effect of angle and speed of droplet release, drop size, and air speed are important initial conditions of the formation of the water cloud and final water distribution. Some uncertainty is caused by the low Mach number assumption. However, the magnitude of the error caused by this assumption is assumed to be acceptably small in proportion to other uncertainties related to the initial boundary conditions because, after the impact, the speeds of both the gas and the liquid phase very rapidly slow down to a level at which the assumption holds. The distribution of the accumulated water on the floor turned out to be sensitive to the numerical force term limiter used.

The future developments of the FDS program should include a more detailed analysis of the sensitivity of the results to the numerical parameters, and the possible validation of some sub-models by comparing the results against some published data on high-speed sprays. The FDS code could be tested by simulating some IMPACT tests using the real initial/boundary conditions of the tests. The possibilities of scaling up to the aircraft size should be studied to find the applicability of the model to the real problem.

\section{SUMMARY AND CONCLUSION}

Liquid dispersal phenomena have been studied in the impact test conducted at VTT. The test results have given valuable information on liquid behaviour under impact conditions. This information can be utilised to better understand fuel dispersal under aircraft-impact conditions, and in applying analysis methods for the determination of fuel spread and fire risk following an airplane crash.

The experimental findings indicate that the liquid release starts along the surface quite perpendicularly to the incoming direction of the missile and forms a fairly "flat" splash pattern. The initial discharge speed of the liquid front coming out from the ruptured missile may be much higher than the impact velocity of the missile, but the propagation speed of the spray front decreases rapidly when the distance from the source increases. 
The results of the preliminary simulation show that the FDS program is a tool usable in simulating the two-phase flows involving high-speed droplets provided that the initial conditions (angle and speed of liquid release, droplet size distribution, and air speed) can be specified satisfactory. Given that, the formation of the water cloud and final water distribution is predicted by FDS reasonably well. The future developments of the FDS program should include a possible validation of some sub-models, and more testing of the program using the real boundary conditions of the IMPACT tests. The ultimate goal is to replace the water with jet fuel and let the model predict the fuel spread and fire risk to a nuclear power plant.

\section{REFERENCES}

1. Kärnä, T., Saarenheimo, A. and Tuomala, M., Impact loaded structures. In: SAFIR, The Finnish Research Programme on Nuclear Power Plants Safety, Interim Report, VTT 2272, Espoo 2004, pp. 113-122.

2. Lastunen, A., Hakola, I., Järvinen, E., Calonius, K., and Hyvärinen, J., "Impact Test Facility,” Paper submitted for SMiRT 2007.

3. Saarenheimo, A., Hakola, I., Hyvärinen, J., and Tuomala, M., "Numerical and Experimental Studies on Impact Loaded Concrete Structures," Proc., of ICONE 14, International Conference on Nuclear Engineering, ICONE1489477, Miami, USA, July 17-20, 2006.

4. Saarenheimo, A., Tuomala, M., Calonius, K., Lastunen, A., Myllymäki, J., and Hyvärinen, J., "Numerical Studies of Impact Loaded Reinforced Concrete Walls," Paper submitted for SMiRT 2007.

5. Silde, A., Hostikka, S., and Kankkunen, A., Study of Liquid Dispersal in IMPACT Tests: Summary Report, VTT Research Report VTT-R-12084-06, February 2007.

6. McGrattan, K., (ed.) Fire Dynamics Simulator (Version 4) - Technical Reference Guide, Special Publication 1018, National Institute of Standards and Technology, Gaithersburg, MD, 94 p. 